\title{
Beyond "Age": Frailty Assessment Strategies Improve Care of Older Patients with Cancer
}

\author{
Riccardo A. Audisio, MD, FRCS ${ }^{1}$ and Barbara L. van Leeuwen, MD, $\mathrm{PhD}^{2}$ \\ ${ }^{1}$ St Helens Teaching Hospital, University of Liverpool, St Helens, UK; ${ }^{2}$ Department of Surgery, University Medical Center \\ Groningen, Groningen, The Netherlands
}

The progress in scientific and medical knowledge has been fascinating over the last few decades and has had a noticeable impact on the most recent clinical practice. However, not every patient benefits from this progress in the same way; European and North American data are there to prove how senior patients with cancer are offered a substandard treatment, predominantly surgical treatment.

The majority of cures for solid tumors results from local treatment, especially from surgery (approximately $60 \%$ ) and radiation therapy (approximately $40 \%$ ). ${ }^{1}$ In as much as surgical oncologists can be proud of curing the largest part of cancer patients, they also need to take responsibility for their failures: most noticeably, there is abundant evidence to show that older cancer patients have a far worse outcome. $^{2}$ This is usually a result of under treatment, but could also result from inappropriate overtreatment. Inferior cancer-related survival is often a fault that lies in surgery, rather than a fault due to all other treatments combined.

Personalized treatment should entail the offer of a treatment option that has been specifically tailored to every individual patient - not one roused solely from a biologically targeted treatment plan. Now is the time when we must accept that the "eye-ball test" is neither an effective nor precise enough option.

Despite the fact that clinical experience allows a wise surgeon to identify (to some extent) the patients that need to be brought to theater and those who do not, any superficial appraisal of the patient fitness leads to an inaccurate assessment. It also affects the consenting process;

(C) Society of Surgical Oncology 2015

First Received: 6 July 2015;

Published Online: 5 August 2015

R. A. Audisio, MD, FRCS

e-mail: raudisio@doctors.org.uk disallowing the comparison between results of treatment in different units, and it prevents older patients from being entered into clinical trials. Even world-renowned centers of excellence omit data on frailty, thus failing to identify which patients have been involved in their studies. Many studies on elderly cancer patients only include age and ASA to determine their patients' performance, with no reference to comorbidities, frailty, or performance status. Consequently, these studies present highly biased findings retrieved from super-selected cohorts, whose conclusions cannot be applied to the overall geriatric population as the most prominent in frequency. Furthermore, the focus of outcome studies in elderly surgical patients is often on short-term outcomes, and although this is important in order to achieve a true "shared" decision, a patient ought to be informed on the long-term outcomes of a surgical procedure as well. In this sense, quality is often more important than quantity in the elderly age group.

It would be totally inappropriate to analyze clinical findings and surgical outcomes without clearly stating patients' stage - on this much I think we can all agree. Thus the TNM staging system is a fundamental tool in drafting treatment plans, comparing series, testing new treatments, and drafting guidelines. The first version of the TNM staging system was introduced in the 1950s to resolve this problem, but it was not a perfect tool; it was closely monitored, amended, and improved. At this moment in time, we are relying on the existing implementation of the seventh edition, while we wait for the eighth to be introduced.

In a similar way, it is inappropriate to present a series of older patients without disclosing frailty data-age alone is not enough to take an accurate snapshot of the general health condition presented. Although we have yet been unable to design the perfect tool for this purpose, two decades of discussions with geriatricians have provided us 
with an understanding of just how valuable geriatric assessment is, as a vital instrument in the decision-making process-it is essential. Up to this point, geriatric assessment has been tested and validated to show that it can indeed allow for a prediction of survival probability and operative risks including postoperative delirium. ${ }^{3-8}$

This issue of Annals of Surgical Oncology presents a scientific article by Dr. M. D. Sur and coworkers investigating the use of radiographic preoperative assessment of sarcopenia (by means of estimates of psoas muscle volume and density). ${ }^{9}$ We praise these authors for analyzing new ways to enhance the assessment process, by including sarcopenia into the equation through this novel approach and integrating it with the presence of self-reported exhaustion. This combined approach is proving to be rather accurate in identifying a frail subset of older patients undergoing pancreaticoduodenectomy for pancreatic cancer. The information that is reported is welcome in view of validating its use on prospective future series of older patients undergoing cancer surgery, although the cause of sarcopenia and its relation to malnutrition are not discussed in this paper. It is known that malnutrition is an important predictive factor of adverse outcome and very likely that an accurate measurement of sarcopenia is the most precise way to define malnourishment, which is so common among older individuals $(50 \%){ }^{6}$ The inferior outcomes in sarcopenic patients prompt the authors to underscore the need for prehabilitation. Such interventions show great promise and could potentially improve surgical outcomes. The debate is ongoing, however-if sarcopenia is to be viewed as a sign of tumor aggression or a sign of frailty is yet to be determined. The question remains: should we take the time to improve a patients' nutritional and functional status preoperatively or should we take the tumor out as soon as possible? In light of this example, many questions still need to be answered on the road to determine a truly personalized approach to medicine, and, at this time, geriatric assessment is an invaluable beacon to light this way.

\section{REFERENCES}

1. Morgan G, Ward R, Barton M. The contribution of cytotoxic chemotherapy to 5-year survival in adult malignancies. Clin Oncol. 2004;16:549-60.

2. De Angelis R, Sant M, Coleman MP, Francisci S, Baili P, Pierannunzio D, et al. EUROCARE-5 Working Group. Cancer survival in Europe 1999-2007 by country and age: results of EUROCARE-5-a population-based study. Lancet Oncol. 2014; 15:23-34.

3. Wildiers H, Heeren P, Puts M, Topinkova E, Janssen-Heijnen ML, Extermann M, et al. International Society of Geriatric Oncology consensus on geriatric assessment in older patients with cancer. J Clin Oncol. 2014;32:2595-603.

4. Korc-Grodzicki B, Downey RJ, Shahrokni A, Patel SG, Audisio R. Surgical considerations in older adults with cancer. J Clin Oncol. 2014;32:2647-53. doi:10.1200/JCO.2014.55.0962.

5. Audisio RA, Pope D, Ramesh HS, Gennari R, van Leeuwen BL, West C, et al. PACE participants. Shall we operate? Preoperative assessment in elderly cancer patients (PACE) can help. A SIOG surgical task force prospective study. Crit Rev Oncol Hematol. 2008;65:156-63.

6. Huisman MG, Audisio RA, Ugolini G, Montroni I, Vigano A, et al. Screening for predictors of adverse outcome in onco-geriatric surgical patients: a multicenter prospective cohort study. Eur J Surg Oncol. 2015;41:844-51.

7. Huisman MG, van Leeuwen BL, Ugolini G, Montroni I, Spiliotis J, Stabilini C, et al. "Timed Up \& Go": a screening tool for predicting 30-day morbidity in onco-geriatric surgical patients? A multicenter cohort study. PLoS One. 2014;9:e86863.

8. Korc-Grodzicki B, Sun SW, Zhou Q, Iasonos A, Lu B, Root JC, et al. Geriatric assessment as a predictor of delirium and other outcomes in elderly patients with cancer. Ann Surg. 2014;261: 1085-90.

9. Sur MD, Namm JP, Hemmerich JA, Buschmann MM, et al. Radiographic sarcopenia and self-reported exhaustion independently predict NSQIP serious complications after pancreaticoduodenectomy in older adults. Ann Surg Oncol. 2015. doi:10.1245/s10434-0154763-1. 